\title{
The Impact of Stress Among Thai Medical Students in Different Years on Mental Health Disorders
}

\author{
Phiraphol Yingjamsiri ${ }^{1}$, Pichamol Imaroonrak ${ }^{2}$, Sirilak Rattanaboonchot ${ }^{3}$, Supapit Pongsawat ${ }^{4}$, Arissara \\ Phrongkhunthod $^{5}$
}

\author{
${ }^{1}$ Triam Udom Suksa School, Bangkok, Thailand \\ ${ }^{2}$ Rattanaburi School, Surin, Thailand \\ ${ }^{3}$ Samakkhiwittayakhom School, Chiang Rai, Thailand \\ ${ }^{4}$ Pakthongchai Prachaniramit School, Nakhon Ratchasima, Thailand \\ ${ }^{5}$ Maryvit Pattaya School, Chon Buri, Thailand \\ DOI: 10.29322/IJSRP.11.07.2021.p11589 \\ http://dx.doi.org/10.29322/IJSRP.11.07.2021.p11589
}

\begin{abstract}
In modern times, teens and young adults are generally known to be "stressed" more than they were in the past. Many people believe that stress is a distant thing, but in reality, it affects every human being at every stage of life. This can be caused by a variety of factors, such as the family, the environment, and society. Although stress can keep people alert and make them more self-aware, stress can also be a source of distress as well. Based on these observations, an online survey of ten questions was used to conduct a study of the impact of stress on the target group, medical students. The study involves 97 participants who are medical students currently studying between years 1 to year 6 from various universities in Thailand and have encountered stress during the academic years of the medical program. The objective of this research is to understand the nature of stress so everyone can be aware and deal with it and be a guide for students who are intended to study in the medical faculty. The results were divided into three different types, composed of acute stress, episodic acute stress, and chronic stress. This has led to the conclusion that medical students are more likely to experience episodic acute stress more than acute stress or chronic stress, and their stress level increases as the medical program continues. According to these results, other preventive measures can be further conducted to prevent medical students from suffering a high level of stress which might lead to mental health disorders such as depression, anxiety, migraine headaches, and other mental issues.
\end{abstract}

Index Terms- Distress, Thai Medical Students, Self-awareness, Mental Health Disorders

\section{INTRODUCTION}

"Stress" is a short word that every individual encounters in human life. Stress can be defined as "a condition or feeling experienced when a person perceives that the demands placed on them exceed the resources the individual has available" (What is Stress?, 2017). Nowadays, we have found that stress has become a major problem. This can be seen from the news around us from several media today, for instance, suicidal news of people with high-stress levels.

Undergraduates in tertiary education are a group of people who are growing toward adulthood. They have to get good preparation for their long life, including their academic life, social adjustment, daily activities, and also their responsibilities. All of these factors may lead them to face stress in many aspects. Moreover, previous studies have shown that medical students have been reported to suffer from higher perceived stress compared to the general population and students in other academic fields (Heinen et al., 2017). In many medical schools, the environment itself is an all-prevailing pressure situation, providing an authoritarian and rigid system, one that encourages competition rather than cooperation between learners (Styles WM, 1993). Before becoming medical students, they have to work hard with good preparation for 12 years at primary and secondary levels. They also have to be highly selfdisciplined to achieve their goals of becoming medical students. Furthermore, when they get to the campus, they have to prepare themselves harder for the courses of medicine for at least 6 years. As a result, their mental health can also be severely impacted throughout their medical program.

Medical students will study at 2 levels, including preclinical and clinical levels. Preclinical students will learn the details of how both the healthy body and the diseased body functions. They learn hands-on in the laboratory settings, from dissecting the intricacies of the human body in the gross anatomy lab, viewing normal microscope slides in histology and diseased specimens in pathology (preclinical and clinical year). In contrast, clinical students will move from studying in a classroom into a hospital setting. This means that they have to work harder and also interact with real patients. Learning in the medical field is extremely important because this field is related to human lives. Medical students need to be simultaneously learned, honest, kind, humble, enthusiastic, 
optimistic, efficient, careful, flexible, and responsible. They have to control themselves all the time. If not, it may lead to someone's death.

This research will study the nature of stress, the tendency of stress levels, as well as the risk conditions between preclinical and clinical students. The objective is to get more information or facts and to describe the social phenomena that occur in these two target groups. This research will be the basic information for understanding humans and will be useful for guidance work or additional psychological counseling.

\section{METHODOLOGY}

This cross-section study will be conducted by a survey. The survey contains 10 questions to classify the participants who have experienced stress into 3 types of stress, including acute stress, episodic acute stress, and chronic stress. It was blindly distributed as an online survey to medical students in Thailand. There were a total of 97 responses after collecting the results. The researchers use descriptive and inferential statistics to analyze the data. Their answers were evaluated to reveal the different levels of stress between preclinical students and clinical students. Medical students' view toward the influence of the three forms of stress was shown as a mean score from 5 Point Likert scaling responses, ranging from acute stress to chronic stress based on their answers. The results were displayed using a frequency table according to the stress levels of medical undergraduates in different years. The results will be divided into 3 types of stress.

Acute stress (score from 1 to 16) - It is the most common type of stress, in which you can feel this kind of stress when you give speeches in front of the class or in other situations such as seeing a car accident. Acute stress is a short-term effect; the most common symptoms are headache, back pain, diarrhea, high blood pressure, and some combination of anger or irritability. Acute stress symptoms develop quickly over minutes or hours. This type of stress is not so harmful to your body; it is good for your body to release the stress hormones to help your mind and body deal with situations.

Episodic acute stress (score from 17 to 33) - People who live with a repeated stimulus of stress or people who have experienced acute stress before will considered to have episodic acute stress. The symptoms are similar to acute stress, but due to the extended and frequent over-arousal. The common signs and symptoms are depression, sweaty palms, migraine headaches, and asthma.

Chronic stress (score from 34 to 50) - This is the most harmful type of stress. If left for a long period, it may damage your physical and mental health. The symptoms of chronic stress can cause serious damage and can eventually result in physical and mental breakdown. Furthermore, this can lead to death, such as suicide caused by being overstressed, heart attacks, and strokes.

\section{RESULTS}

\section{Preclinical Students}

The numbers in Table 1 and Table 2 represents the level of stress in Thai medical preclinical students.

Table 1: The overview score of stress in preclinical students

\begin{tabular}{|l|c|c|c|}
\hline & $\min$ & $\mathbf{m a x}$ & mean \\
\hline 1st year & 11 & 50 & 22 \\
\hline 2nd year & 16 & 50 & 22 \\
\hline 3rd year & 22 & 22 & 29 \\
\hline
\end{tabular}

$($ Overall mean $=26)$

Table 2: Percentage of preclinical students experiencing different types of stress

\begin{tabular}{|c|c|c|}
\hline Types & Frequency & Percent \\
\hline Acute stress & 5 & 8.77 \\
\hline Episodic acute stress & 40 & 70.18 \\
\hline
\end{tabular}

This publication is licensed under Creative Commons Attribution CC BY. 


\begin{tabular}{|c|c|c|}
\hline Chronic stress & 12 & 21.05 \\
\hline
\end{tabular}

From Table 2, it is noticeable that the percentage of episodic acute stress in preclinical students is the highest among the types of stress, 70.18 percent.

\section{Clinical Students}

The numbers in Table 3 and Table 4 represents the level of stress in Thai medical clinical students.

Table 3: The overview score of stress in clinical students

\begin{tabular}{|c|c|c|c|}
\hline & $\min$ & $\max$ & mean \\
\hline 4th year & 5 & 37 & 24 \\
\hline 5th year & 19 & 46 & 31 \\
\hline 6th year & 32 & 37 & 35 \\
\hline
\end{tabular}

$($ Overall mean $=30)$

Table 4: Percentage of clinical students experiencing different types of stress

\begin{tabular}{|c|c|c|}
\hline Types & Frequency & Percent \\
\hline Acute stress & 2 & 5.88 \\
\hline Episodic acute stress & 25 & 73.53 \\
\hline Chronic stress & 7 & 20.59 \\
\hline
\end{tabular}

From Table 4, the results have shown that episodic acute stress has the highest percent in clinical students, 73.53 percent.

According to Table 1, the overview score of stress in preclinical medical students has an average of 26 points, which is considered to be episodic acute stress. Based on Table 2, almost all of the students in the preclinical medical program appeared to have experienced high-stress levels, known as episodic acute stress, while the prevalence of acute stress in preclinical medical students is low, particularly $70.18 \%$ of preclinical students have episodic acute stress followed by chronic stress $(21.05 \%)$ and acute stress $(8.77 \%)$. Turning to Table 3, the results from clinical students showed the average score (mean) of the fourth-, fifth-, and sixth-year students in the program were 24,31 , and 35 points respectively. The mean scores of fourth- and fifth-year students remained in the range of 17 to 33 points, which means that, on average, they experienced episodic acute stress the most, whereas the sixth-year students' mean score remained in chronic stress. In addition, the sixth-year students ranked the highest in terms of the level of stress, 35 points on average, which is the most when compared to other students in different medical years.

\section{DISCUSSION}

Medical education is associated with various pressures and stresses which can lead to high levels of depression, anxiety, and other mental disorders (Moir et al., 2018). It is reasonable that the overall prevalence of depressive symptoms among medical students is higher than that reported in the general population according to other research (Rotenstein et al., 2016). This is because of several factors that all influenced the intensity of the mental ailments: the large workload, the time commitment, and the number of assessments, as well as the pressures of a preclinical and clinical environment (Sreeramareddy et al., 2007). judged.

The survey was blinded on purpose so that the participants were encouraged to answer truthfully without the hesitation of being

A total of ninety-eight Thai medical students were asked to join the survey and answered all the questions. The research was carried out to assess the amount and level of stress amongst preclinical (year 1 to 3) and clinical (year 4 to 6) medical students. Furthermore, the results from the survey will be analyzed and compared between each medical year. The severity of stress symptoms, including your emotions, behaviors, thinking ability, and physical health, are taken into consideration when classifying medical students into three types of stress (Tan et al., 2018). The levels of stress can be assessed into three categories, composed of acute stress, episodic acute stress, and chronic stress. 
Throughout the course of the six-year medical program, results show that the ubiquity of all three levels of stress is found in both preclinical years and clinical years. The prevalence of stress is noticeable in the study, from the survey recorded from the stressed medical students. According to the survey taken, results have shown that there are three levels of stress occurring throughout every year of medical training. In comparison with the clinical years of medical undergraduates, the average amount of stress in preclinical years is moderately lower than that in the clinical years. As the medical training continued, in the later years of the medical program, there was a significant increase in the percentage of the members with two levels of stress: acute stress and episodic acute stress. The results indicate that as the preparation to become a doctor has continued, the intensity of the medical studies and Thai education has relatively become more extreme. Surprisingly, the results designate that preclinical medical students have more students with chronic stress than their seniors, clinical medical students.

Therefore, it is extremely significant to beware and aware of the impact of stress, especially about the higher levels of stress, chronic stress, as the consequence of it can be a lot more detrimental and serious to people's mental health and wellbeing. Both preclinical and clinical medical students should be concerned about the comparatively high amount of stress they experienced during their medical program, especially those who experienced high levels of stress. Students who have higher levels of stress are more likely to report suicidal thinking more than the ones with fewer stress levels (Rosiek et al., 2016). Additionally, preventive actions should also be applied effectively and efficiently. If preventive measures are taken to decrease the amount of stress among medical students, especially among clinical medical undergraduates who comparably have a higher percentage of stress, then the number of stressed medical students will surely be reduced. In addition, suicidal cases and individuals with mental illness will become smaller in number as well. For instance, social support from different sources, families, friends, and school personnel, can prevent high-stress levels as well as excessive low-stress levels (Ozbay et al., 2007).

\section{CONCLUSION}

The objective is to identify the level of stress present in medical students, undergraduates in academic year $1-6$ from universities in Thailand. A total of 97 people were divided into preclinical and clinical years. The results show that medical students from both groups are most likely at risk of episodic acute stress (on an average of 71.86\%), followed by chronic stress (on an average of $20.82 \%$ ), and acute stress (average of $7.33 \%$ ). This means that medical students tend to encounter symptoms of episodic acute stress more than acute stress or chronic stress. Interestingly, acute stress is present in preclinical students more than in clinical students, while episodic stress is more prevalent in clinical undergraduates than in preclinical undergraduates. Therefore, stress must not be ignored and should be wary to prevent stress at any levels which could be critical to our life and eventually lead to such mental disorders, especially in medical students who are at risk of receiving more stress while studying in college.

\section{REFERENCES}

[1] Heinen, I., Bullinger, M., \& Kocalevent, R.-D. (2017). Perceived stress in first year medical students - associations with personal resources and emotional distress. BMC Medical Education, 17(1).

[2] Moir, F., Yielder, J., Sanson, J., \& Chen, Y. (2018). Depression in medical students: current insights. Advances in medical education and practice, 9, $323-333$.

[3] Ozbay, F., Johnson, D. C., Dimoulas, E., Morgan, C. A., Charney, D., \& Southwick, S. (2007). Social support and resilience to stress: from neurobiology to clinical practice. Psychiatry (Edgmont (Pa. : Township)), 4(5), 35-40.

[4] Preclinical and Clinical Years. (n.d.). Retrieved May 9, 2020, from https://www.umc.edu/som/Students/Current Students/Curriculum/Preclinical and Clinical Years.html

[5] Rosiek, A., Rosiek-Kryszewska, A., Leksowski, Ł., \& Leksowski, K. (2016). Chronic Stress and Suicidal Thinking Among Medical Students. International journal of environmental research and public health, 13(2), 212.

[6] Rotenstein, L. S., Ramos, M. A., Torre, M., Segal, J. B., Peluso, M. J., Guille, C., Sen, S., \& Mata, D. A. (2016). Prevalence of Depression, Depressive Symptoms, and Suicidal Ideation Among Medical Students: A Systematic Review and Meta-Analysis. JAMA, 316(21), $2214-2236$.

[7] Selye, Hans (1956). The Stress of Life. New York: McGraw-Hill.Discussed in Mark Jackson, "The Pursuit of Happiness: The Social and Scientific Origins of Hans Selye's Natural Philosophy of Life", History of the Human Sciences 25/2 (Dec. 2012): 13-29.

[8] Sreeramareddy, C. T., Shankar, P. R., Binu, V. S., Mukhopadhyay, C., Ray, B., \& Menezes, R. G. (2007). Psychological morbidity, sources of stress and coping strategies among undergraduate medical students of Nepal. BMC medical education, 7, 26.

[9] Styles WM. Stress in undergraduate medical education: 'the mask of relaxed brilliance' Br J Gen Pract. 1993;43:46-7.

[10] Tan, S. Y., \& Yip, A. (2018). Hans Selye (1907-1982): Founder of the stress theory. Singapore medical journal, 59(4), $170-171$.

[11] What is Stress? (2017, January 4). Retrieved May 9, 2020, from http://www.stress.org/what-is-stress/

This publication is licensed under Creative Commons Attribution CC BY.

http://dx.doi.org/10.29322/IJSRP.11.07.2021.p11589

WWW.ijsrp.org 